\title{
Nystagmus, CTCAE 5.0
}

National Cancer Institute

\section{Source}

National Cancer Institute. Nystagmus, CT CAE 5.0. NCI Thesaurus. Code C146775.

A disorder characterized by involuntary movements of the eyeballs. 